\title{
(2) OPEN ACCESS \\ COVID-19 aerosol box as protection from droplet and aerosol contaminations in healthcare workers performing airway intubation: a randomised cross- over simulation study
}

\author{
Muhaimin Noor Azhar, ${ }_{1}^{1}$ Aida Bustam 다, 'Khadijah Poh, ${ }^{1}$ \\ Ahmad Zulkarnain Ahmad Zahedi, ${ }^{1}$ Mohd Zahir Amin Mohd Nazri, ${ }^{1}$ \\ Mohammad Aizuddin Azizah Ariffin, ${ }^{1}$ Mohd Hafyzuddin Md Yusuf, ${ }^{1}$ Aliyah Zambri, ${ }^{1}$ \\ Johnathan Y O Chong, ${ }^{2}$ Anhar Kamarudin, ${ }^{1}$ Bin Ting Ang, ${ }^{2}$ Affan Iskandar, ${ }^{2}$ \\ Keng Sheng $\mathrm{Chew}^{3}$
}

Handling editor Katie Walker

- Additional material is published online only. To view please visit the journal online (http://dx.doi.org/10.1136/ emermed-2020-210514).

${ }^{1}$ Academic Unit of Emergency Medicine, Faculty of Medicine, University of Malaya, Kuala Lumpur, Malaysia

${ }^{2}$ Department of Emergency Medicine, University of Malaya Medical Centre, Kuala Lumpur, Malaysia

${ }^{3}$ Faculty of Medicine and Health Sciences, University of Malaysia Sarawak, Kota Samarahan, Malaysia

\section{Correspondence to}

Dr Aida Bustam, Academic Unit of Emergency Medicine, Faculty of Medicine, University of Malaya, Kuala Lumpur 50603, Malaysia;

aidabustam@um.edu.my

Received 10 August 2020 Revised 16 October 2020 Accepted 19 October 2020 Published Online First 20 November 2020

\section{Linked}

- http://dx.doi.org/10.1136/ emermed-2020-210761

Check for updates

(C) Author(s) (or their employer(s)) 2021. Re-use permitted under CC BY-NC. No commercial re-use. See rights and permissions. Published by BMJ.

To cite: Noor Azhar $\mathrm{M}$,

Bustam A, Poh K

et al. Emerg Med J

2021:38:111-117.

\section{ABSTRACT}

Background Concerns over high transmission risk of SARS-CoV-2 have led to innovation and usage of an aerosol box to protect healthcare workers during airway intubation in patients with COVID-19. Its efficacy as a barrier protection in addition to the use of a standard personal protective equipment (PPE) is not fully known. We performed a simulated study to investigate the relationship between aerosol box usage during intubation and contaminations on healthcare workers pre-doffing and post-doffing of PPE.

Methods This was a randomised cross-over study conducted between 9 April to 5 May 2020 in the ED of University Malaya Medical Centre. Postgraduate Emergency Medicine trainees performed video laryngoscope-assisted intubation on an airway manikin with and without an aerosol box in a random order. Contamination was simulated by nebulised Glo Germ. Primary outcome was number of contaminated front and back body regions pre-doffing and post-doffing of PPE of the intubator and assistant. Secondary outcomes were intubation time, Cormack-Lehane score, number of intubation attempts and participants' feedback.

Results Thirty-six trainees completed the study interventions. The number of contaminated front and back body regions pre-doffing of PPE was significantly higher without the aerosol box (all $p$ values $<0.001$ ). However, there was no significant difference in the number of contaminations post-doffing of PPE between using and not using the aerosol box, with a median contamination of zero. Intubation time was longer with the aerosol box ( 42.5 s vs $35.5 s, p<0.001)$. CormackLehane scores were similar with and without the aerosol box. First-pass intubation success rate was $94.4 \%$ and $100 \%$ with and without the aerosol box, respectively. More participants reported reduced mobility and visibility when intubating with the aerosol box.

Conclusions An aerosol box may significantly reduce exposure to contaminations but with increased intubation time and reduced operator's mobility and visibility. Furthermore, the difference in degree of contamination between using and not using an aerosol box could be offset by proper doffing of PPE.

\section{Key messages}

What is already known about this subject

- The aerosol box was conceived during the COVID-19 pandemic to protect healthcare workers from exposure to viral contamination during aerosol generating procedures.

- Studies investigating its efficacy in reducing the amount of contaminations on healthcare workers are currently limited to small studies, or studies that measured airborne particles and not contaminations on healthcare workers.

What this study adds

- In this randomised cross-over study simulating aerosolisation during intubation, the aerosol box reduced droplet and aerosol contaminations on healthcare intubators personal protective equipment (PPE). However, post-doffing contaminations were similarly low between aerosol box and no aerosol box usage.

- The increased intubation time and restricted movement when using the aerosol box in our study corroborate prior research.

- The aerosol box should be used with caution. Clinicians should consider patient safety risks particularly in difficult airway or severely hypoxic patients, since contamination can be mitigated by proper donning and doffing of PPE.

\section{BACKGROUND}

Since the outbreak of the novel coronavirus disease (COVID-19) in December 2019, healthcare workers around the world have been concerned about its high risk of transmission. COVID-19, caused by SARS-CoV-2, is transmitted primarily through respiratory droplets. ${ }^{1}$ Appropriate use of personal protective equipment (PPE) can significantly reduce the risk of transmission. ${ }^{2}$ However, interventions such as airway intubation and mechanical ventilation can potentially generate aerosols of viruscontaminated respiratory secretions. ${ }^{34}$ Aerosols can 
remain suspended in the air for prolonged periods and traverse larger distances, thus increasing the risk of transmission. ${ }^{5-7}$

Cases and deaths worldwide have continued to rise, and healthcare workers have been infected. ${ }^{8}$ In the hopes of protecting healthcare workers while performing an intubation, a Taiwanese anaesthetist (HY Lai) designed a transparent plastic box known as the 'aerosol box' that can be placed over a patient's head. ${ }^{10}$ This box potentially enables physicians to intubate patients while shielding themselves from exposure to viral contamination. Nevertheless, the use of such device may add another layer of complexity to performing airway intubation. ${ }^{11}$ Furthermore, its benefit as a barrier protection in addition to the use of a standard PPE is not fully known.

A simulation study by Canelli et $a l^{12}$ using fluorescent dye demonstrated the aerosol box reduced contamination on the intubator. However, this was a small study $(n=2)$ and the authors noted that their simulation produced more droplets than aerosols. To further investigate the efficacy of an aerosol box as an additional barrier protection against aerosol and droplet contaminations, we performed a randomised cross-over simulation study to compare the degree of contamination on the intubator and assistant with and without the use of an aerosol box during airway intubation on a manikin.

\section{METHODS}

\section{Study design, setting and participants}

The randomised cross-over study was conducted from 9 April to 5 May 2020 at the ED of University Malaya Medical Centre (UMMC). UMMC is a university hospital in Kuala Lumpur, Malaysia, designated for screening and admission of patient with COVID-19 during the coronavirus pandemic. Participants were year 1 to year 4 postgraduate emergency medicine trainees. Enrolment was voluntary and all recruited trainees signed an informed consent form.

Participants were randomly allocated to sequence 'AB' (intubation without aerosol box and then with aerosol box) and 'BA' (intubation with aerosol box and then without aerosol box) using computer-generated random numbers, block stratified by year of postgraduate training. One random allocation sequence was generated to assign the trainees as 'intubator' and another sequence as 'assistant'. A person not directly involved in the study procedure carried out the intervention assignment to the trainees according to the random allocation sequence. The crossover design had several advantages over a parallel-group design: it allowed each participant to serve as his or her own matched control, therefore reducing the within-subject variations; smaller sample size would be required to detect meaningful effect at the same level of statistical power as a parallel design; and participants in this cross-over trial could express their preferences by comparing their experiences of the two interventions. ${ }^{13} 14$

\section{Interventions}

All participants wore PPE in accordance to our hospital guideline for performing aerosol generating procedures in the management of patients with COVID-19. The PPE consisted of a mask (surgical mask was used instead of N95 mask due to concerns for costs and availability), a liquid and particle protection coverall with hood, a face shield, shoe covers, a plastic apron and double layered gloves. Verbal instruction for the steps of donning and doffing of PPE was provided by a staff (blinded to the interventions) with the aid of a video.

The aerosol box used in this study (figure 1) was a modified version of the original design by Lai. ${ }^{10}$ Each arm aperture was

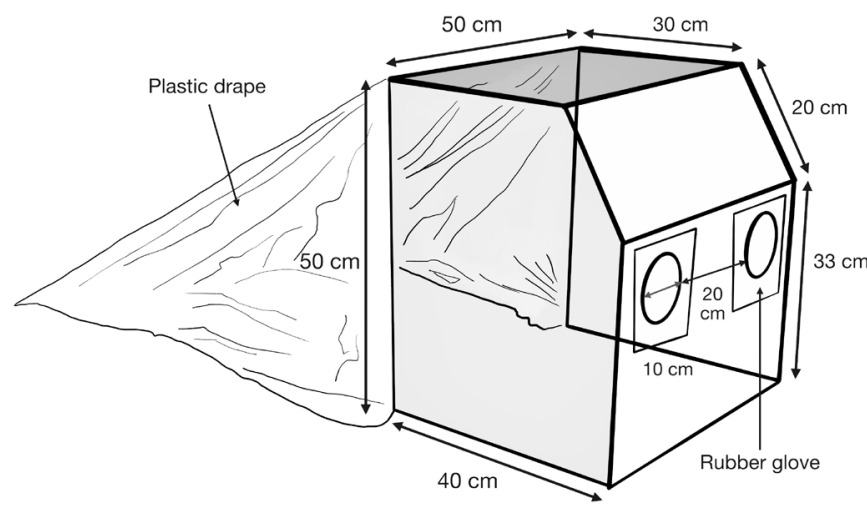

Figure 1 The aerosol box design and dimensions.

covered with a rubber glove that was cut into a square shape and secured with transparent parcel tape. A small hole was cut in the centre of the rubber glove barrier for access and tight seal around the intubator's arms. A transparent plastic sheet was attached to the box to be draped over the patient's (manikin) chest to limit dispersion of aerosols.

The study was performed in a patient bay in the ED. An airway manikin (Laerdal Airway Management Trainer) was placed on a trolley bed. The bed height was adjusted to the intubator's preference prior to the procedure start time. The role of the assistant was to help in handling the intubation equipment from the right side of the manikin. Intubation equipment included a C-MAC (KARL STORZ) video laryngoscope with a size 4 Macintosh blade, a standard adult size bag-valve-mask, a size 7 endotracheal tube with a stylet in-situ, a PEEP valve, and a virus filter (online supplemental figure 1A). Prior to the start of the interventions, each participant had the opportunity to practice performing the video laryngoscope-assisted intubation with and without the aerosol box in the same simulation setting.

To simulate aerosolised contaminants, we used a single-use nebuliser chamber filled with $10 \mathrm{~mL}$ of Glo Germ solution (Moad, Utah) (online supplemental figure 1B). Glo Germ contains $1-5 \mu \mathrm{m}$ melamine copolymer resin beads in mineral oil and can be visualised under ultraviolet A (UVA) light in darkened room. It has been used as surrogate measures of airway aerosol contaminants during intubation. ${ }^{15}$ The nebuliser chamber was taped near the manikin's right angle of mouth and the air flow to the nebuliser was set to $5 \mathrm{~L} / \mathrm{min}$.

The intubator was first asked to place the bag-valve mask on the manikin to simulate preoxygenation. The intubator then removed the bag-valve mask and intubated the manikin. The intubator rated the Cormack-Lehane view. One of the study investigators (AZAZ), independently assessed the video laryngoscope Cormack-Lehane (VL-CL; assessor VL-CL) achieved by the intubators as comparison. The intubator was allowed to perform further intubation attempts if needed (without preoxygenation in between), until successful intubation was achieved. The Glo Germ nebulisation was set to flow from the time the intubator introduced the laryngoscope into the manikin's oral cavity until the correctly placed endotracheal tube was connected to the ventilator tubing.

Each participant performed intubation twice (with and without the aerosol box) and performed the role of an assistant twice (with and without the aerosol box). Intervention periods were separated by an interval of about 2 hours to allow adequate time for cleaning of equipment and the room. No carry over effect was expected from the interventions. 


\section{Outcome measures}

The primary outcome measure was number of body regions contaminated. Measurement of contamination was performed by MZAMN, MAAA, MHMY, JYOC and BTA (who were blinded to the interventions), in a clean area immediately after the participants had completed the intubation procedure. Body regions that contained any fluorescent stains of any size under UVA light were considered as contaminated (online supplemental figure 1C). Body regions were divided into 15 regions on the front and 15 regions on the back (online supplemental figure 2). Eight comparisons for the primary outcome measure were performed (pre-doffing and post-doffing contaminations of the front regions and the back regions for the intubator and the assistant). Secondary outcome measures were frequency of contamination by body regions, VL-CL score, time to successful intubation (from the removal of bag-valve mask until correctly placed endotracheal tube was connected to the ventilator tubing), number of intubation attempts, room contamination radius (defined as the furthest floor contamination distance from the nebuliser chamber), and participants' feedback using a 5-point Likert scale rating on handling of equipment, visibility (Cormack-Lehane view) during performing the procedure, confidence in achieving successful intubation, degree of movement, and perception of risk of exposure to contamination.

\section{Sample size}

Sample size was calculated using G-power V.3.1.5. (Heine Heinrick University, Düsseldorf, Germany). As we wanted to perform eight comparisons for the primary outcome measure, the alpha value was adjusted to 0.00625 using Bonferroni correction of the significance level to protect against type I error. Based on the findings from Canelli et al, ${ }^{12}$ we estimated an effect size of 0.8 (large effect size ${ }^{16}$ ) to detect a more clinically relevant difference in the number of contaminated body regions.

Using a two-tailed t test for matched pairs, alpha of 0.00625 , beta of 0.05 and an effect size of 0.8 , a total sample of 34 was required. A potential drop out rate of $20 \%$ made the estimated sample size 40 . Our centre has 64 postgraduate emergency medicine trainees and we recruited the first 40 trainees who consented to participate in the study.

\section{Statistical analysis}

Data were analysed using SPSS V.26.0.0.0 for Mac OS. Participant demographic data (age, gender, height, weight, body surface area, dominant hand, years of experience working in ED and postgraduate year of training) were analysed using descriptive statistics. Continuous variables were tested for normality with Shapiro-Wilk test. For the primary and secondary outcome measures, continuous variables were analysed using paired samples $t$ test or Wilcoxon signed rank test depending on the normality of data. Categorical variables were analysed using McNemar and McNemar-Bowker tests. A p value of $<0.00625$ was considered as statistically significant for the primary outcome and a $\mathrm{p}$ value of $<0.05$ was considered as statistically significant for the secondary outcomes.

\section{Patient and public involvement}

Patients or the public were not involved in the design, conduct, reporting or dissemination plans of our research.

\section{RESULTS}

\section{Participants}

Forty participants were recruited into the study. Four participants were excluded after randomisation: one due to unavailability of a suitable coverall size, and three due to inability to attend the study intervention periods (figure 2). A total of 36 participants completed the study interventions. Characteristics of the participants are summarised in table 1 .

\section{Primary outcomes}

The overall contamination results of intubators and assistants, and the primary outcomes are shown in table 2. There were significantly more areas of contamination found on the PPE of intubators and assistants (pre-doffing contaminations) without the aerosol box than with (median of 10.5-13 body regions vs $6-9$ body regions, all p values $<0.001$ ) (table 2 ). However, postdoffing of PPE, there was no significant differences between methods in the number of contamination areas (median of contamination areas was zero for both intubators and assistants). Frequencies and percentages of participants by number of contaminated body regions are provided in online supplemental tables 1 and 2.

\section{Secondary outcomes}

The frequencies of contamination based on body regions of the intubators are presented in figure 3. With the use of the aerosol box, contaminations were most frequently found on the forearms, hands and front torso of the intubators. The use of aerosol box significantly reduced the number of contaminations on the intubators' PPE-protected face, head, neck, arms, thighs, legs and feet. However, post-doffing contaminations were found on the forearms of the intubators more frequently following the use of the aerosol box compared with no aerosol box, although the difference was not statistically significant. Contamination patterns of the assistants were similar to that of the intubators (see online supplemental figure 3 ).

There was no significant difference in VL-CL score reported by the participants when intubating with the aerosol box compared with no aerosol box (table 2). There was no significant difference in the VL-CL score between the assessor and the intubators. Compared with no aerosol box, the use of the aerosol box resulted in longer intubation time $(42.5 \mathrm{~s}$ vs $35.5 \mathrm{~s}, \mathrm{p}<0.001)$, lower first-pass success rate for intubation (94.4\% vs $100 \%)$, and larger room floor contamination radius $(1.62 \pm 0.59 \mathrm{~m}$ vs $1.10 \pm 0.49 \mathrm{~m}, \mathrm{p}<0.001)$.

Participants' subjective rating of their experience is summarised in table 3. More participants reported better experience in intubating without the aerosol box in terms of handling of equipment, visibility, confidence in achieving successful intubation and degree of movement. However, the majority of participants perceived high risk of exposure with no aerosol box.

Correlation analysis yielded no statistically significant correlations between the number of contaminated body regions and participants' age, gender, height, weight, body surface area, dominant hand, years of experience, postgraduate training year and intubation time (online supplemental table 3).

\section{DISCUSSION}

It has been presumed that the aerosol box protects healthcare workers against droplet and airborne contaminations during intubation. Our simulation study found, as expected, that a significantly greater number of body regions were contaminated when performing intubation without the aerosol box 


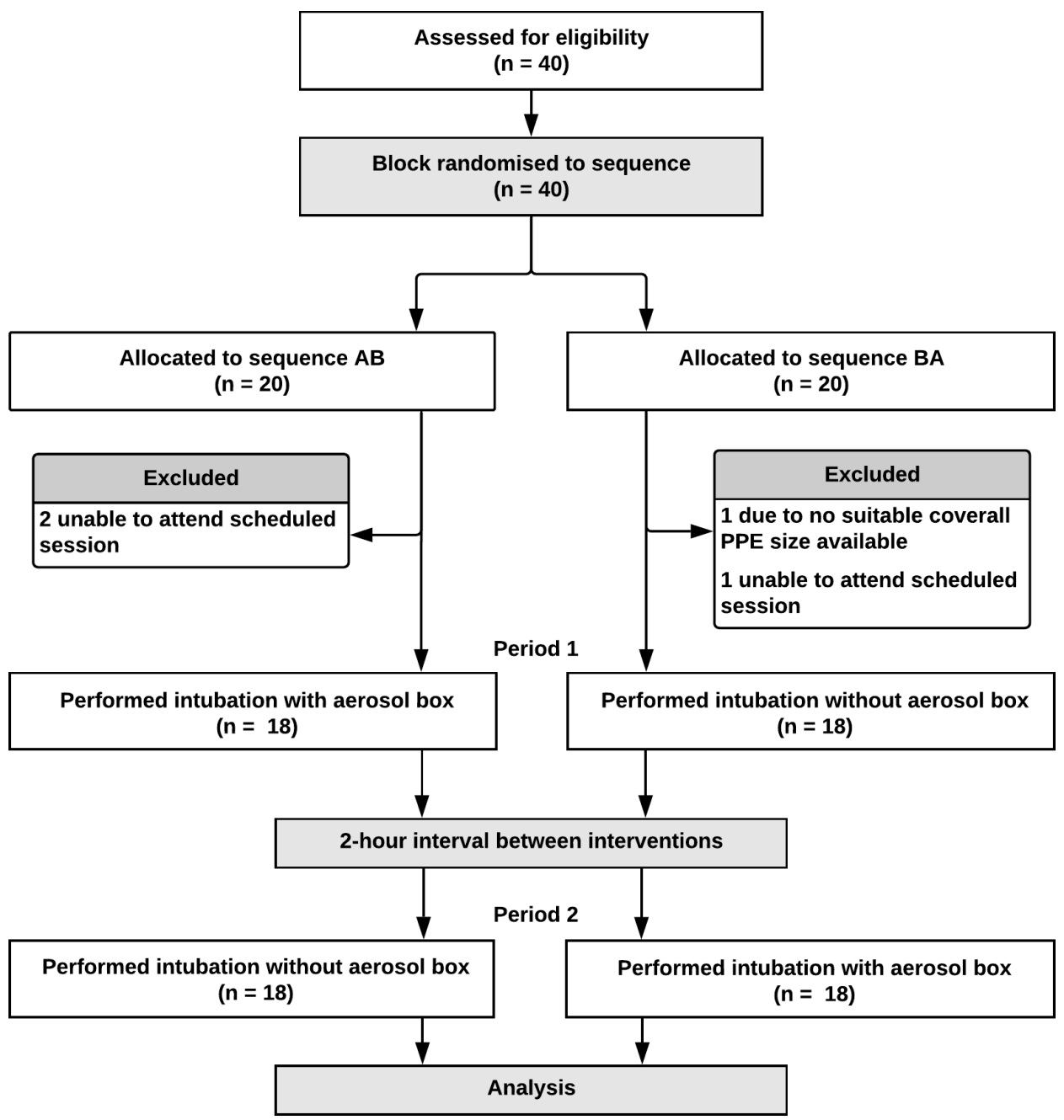

Figure 2 Participant enrolment and randomisation.

(pre-doffing contaminations). However, the study also demonstrated that if PPE donning was done properly and there was no breach of PPE during the procedure, the risk of selfcontamination was reduced. The non-significant difference in post-doffing contaminations between using and not using the aerosol box in our study suggests that the current standard PPE is adequate irrespective of whether additional barrier protection is applied or not. The importance of proper doffing has been

\begin{tabular}{lc}
\hline Table 1 Characteristics of participants & \\
\hline Variables & $\mathrm{N}=36$ \\
\hline Median age, year (IQR) & $33(31-34)$ \\
\hline Male sex, no. (\%) & $11(30.6 \%)$ \\
\hline Mean body weight, kg $( \pm$ SD) & $65.2( \pm 15.0)$ \\
\hline Mean height, cm $( \pm$ SD) & $162.3( \pm 6.7)$ \\
\hline Mean BSA, ${ }^{2}$ & $1.71( \pm 0.22)$ \\
\hline Left-handed, no. $(\%)$ & $6(16.7 \%)$ \\
\hline Mean years in ED, year $( \pm$ SD) & $5.6( \pm 2.0)$ \\
\hline Postgraduate training level, no. $(\%)$ & \\
\hline Year 1 & $16(44.4 \%)$ \\
\hline Year 2 & $7(19.4 \%)$ \\
\hline Year 3 & $3(8.3 \%)$ \\
\hline Year 4 & $10(27.8 \%)$ \\
\hline
\end{tabular}

BSA, body surface area calculated using the Mosteller formula. previously demonstrated, ${ }^{17} 18$ and frequencies and patterns of post-doffing contamination in our study are similar to findings from previous studies investigating risks of self-contamination during PPE donning and doffing. ${ }^{19} 20$

The aerosol box may prevent the splashing of secretions and reduce the dispersion of aerosolised particles during the intubation procedure. This, however, may depend on the aerosol box design, and healthcare workers may still be exposed in the post intubation period especially when the box is removed. A recent study by Simpson et $a l^{21}$ found that the use of the aerosol box resulted in higher airborne particle exposure compared with no device which continued after the device was removed, as measured with a particle counter placed near the laryngoscopist's head. They noted clouds of aerosolised particles escaping through the arm openings of their aerosol box towards the laryngoscopist. The lower contamination rates in our aerosol box intervention suggests that the different design of our aerosol box with covered arm openings might have limited the leak of airborne particles from the box.

Whether greater amounts of contamination on PPE increase the risk of self-contamination during doffing has not been previously explored. In our study, the forearms were noted to be more frequently contaminated post-doffing of PPE when the aerosol box was used compared with no aerosol box. This could be due to a higher risk of self-contamination during doffing given that there would be more contaminants concentrated within the box, 
Table 2 Primary and secondary outcomes

\begin{tabular}{|c|c|c|c|}
\hline Outcomes & $\begin{array}{l}\text { Without aerosol box } \\
(\mathrm{N}=36)\end{array}$ & $\begin{array}{l}\text { With aerosol box } \\
(\mathrm{N}=36)\end{array}$ & $P$ value \\
\hline \multicolumn{4}{|c|}{ Overall no. of contaminated participants* } \\
\hline \multicolumn{4}{|c|}{ Total no. of intubators with contaminations: } \\
\hline Pre-doffing & $36(100.0 \%)$ & $36(100.0 \%)$ & \\
\hline Post-doffing & $13(36.1 \%)$ & $16(44.4 \%)$ & $0.607 \dagger$ \\
\hline \multicolumn{4}{|c|}{ Total no. of assistants with contaminations: } \\
\hline Pre-doffing & $36(100.0 \%)$ & $36(100.0 \%)$ & \\
\hline Post-doffing & $11(30.6 \%)$ & $9(25 \%)$ & $0.791 \dagger$ \\
\hline \multicolumn{4}{|l|}{ Primary outcome } \\
\hline \multicolumn{4}{|l|}{ Number of body regions contaminated: } \\
\hline \multicolumn{4}{|l|}{ Pre-doffing } \\
\hline \multicolumn{4}{|l|}{ Intubator } \\
\hline Front, median (IQR)‡ & $13.0(12.0-15.0)$ & $9.0(7.0-11.0)$ & $<0.001 \S$ \\
\hline Back, median (IQR)‡ & $13.0(12.0-13.0)$ & $6.0(4.0-8.0)$ & $<0.001 \S$ \\
\hline \multicolumn{4}{|l|}{ Assistant } \\
\hline Front, median (IQR)‡ & $12.0(9.0-13.8)$ & $7.0(5.0-9.0)$ & $<0.001 \S$ \\
\hline Back, median (IQR)‡ & $10.5(6.0-13.0)$ & $6.0(4.0-8.0)$ & $<0.001 \S$ \\
\hline \multicolumn{4}{|l|}{ Post-doffing } \\
\hline \multicolumn{4}{|l|}{ Intubator } \\
\hline Front, median (IQR)‡ & $0.0(0.0-1.0)$ & $0.0(0.0-1.0)$ & $0.439 \S$ \\
\hline Back, median (IQR)‡ & $0.0(0.0-0.0)$ & $0.0(0.0-0.0)$ & $1.000 \S$ \\
\hline \multicolumn{4}{|l|}{ Assistant } \\
\hline Front, median (IQR)‡ & $0.0(0.0-1.0)$ & $0.0(0.0-0.0)$ & $0.380 \S$ \\
\hline Back, median (IQR)‡ & $0.0(0.0-0.0)$ & $0.0(0.0-0.0)$ & $0.714 \S$ \\
\hline \multicolumn{4}{|l|}{ Secondary outcomes } \\
\hline \multicolumn{4}{|l|}{ Intubator VL-CL score, no. (\%) } \\
\hline Grade 1 & $19(52.8 \%)$ & $20(55.6 \%)$ & 0.7539 \\
\hline Grade $2 a$ & $8(22.2 \%)$ & $10(27.8 \%)$ & \\
\hline Grade $2 b$ & $8(22.2 \%)$ & $6(16.7 \%)$ & \\
\hline Grade 3 & $1(2.8 \%)$ & 0 & \\
\hline Grade 4 & 0 & 0 & \\
\hline \multicolumn{4}{|l|}{ Assessor VL-CL score, no. (\%) } \\
\hline Grade 1 & $17(47.2 \%)$ & $21(58.3 \%)$ & 0.2739 \\
\hline Grade $2 a$ & $16(44.4 \%)$ & $13(36.1 \%)$ & \\
\hline Grade $2 b$ & $3(8.3 \%)$ & $2(5.6 \%)$ & \\
\hline Grade 3 & 0 & 0 & \\
\hline Grade 4 & 0 & 0 & \\
\hline Median intubation time, $\mathrm{s}$ (IQR) & $35.5(29.3-42.6)$ & $42.5(35.9-47.6)$ & $<0.001 \S$ \\
\hline \multicolumn{4}{|l|}{ Intubation attempts, no. (\%) } \\
\hline 1 & $36(100 \%)$ & $34(94.4 \%)$ & \\
\hline 2 & 0 & $2(5.6 \%)$ & \\
\hline Room contamination radius, $\mathrm{m}( \pm \mathrm{SD})$ & $1.62( \pm 0.59)$ & $1.10( \pm 0.49)$ & $<0.001$ ** \\
\hline
\end{tabular}

Kruskal-Wallis analyses of the difference in video laryngoscope Cormack-Lehane (VL-CL) score between the Assessor and the Intubators yielded no significant difference for both with and without the intubation box ( $p=0.157$ and $p=0.135$, respectively).

*Having at least one body region contaminated.

†Analysis performed with McNemar test.

¥0ut of a total number of 15 body regions for the front and 15 body regions for the back.

$\S$ Analysis performed with Wilcoxon signed rank test (data not normally distributed).

ๆAnalysis performed with McNemar-Bowker test (for VL-CL Grade 1, 2a and 2b).

${ }^{* *}$ Analysis performed with paired $t$ test (data normally distributed).

or due to breaches in PPE as was found in a recent study by Begley et al. $^{22}$ We did not include breach in PPE during intubation procedure as one of the measures in our study. Although this would be an important risk of contamination, it might also confound result analysis as PPE breaches may also happen during doffing. Therefore, measure of contaminations may be a more important outcome.
Despite the intended benefits of an added barrier protection, it is important to consider how the barrier device might affect intubation performance of healthcare workers. A smooth intubation process necessitates having good visibility, adequate room for spatial manipulation of the intubation devices, and the operator's confidence in handling the equipment to perform the procedure. The combination of these factors may influence 
A. Pre-doffing

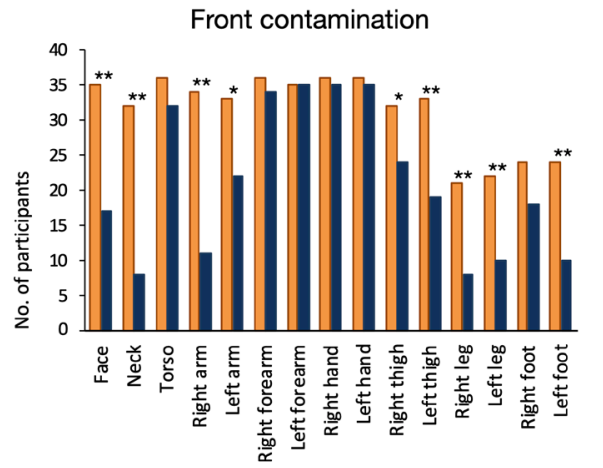

Without aerosol box

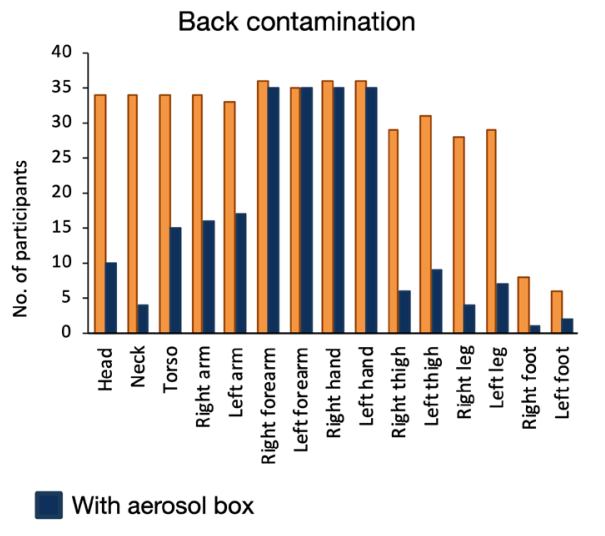

B. Post-doffing

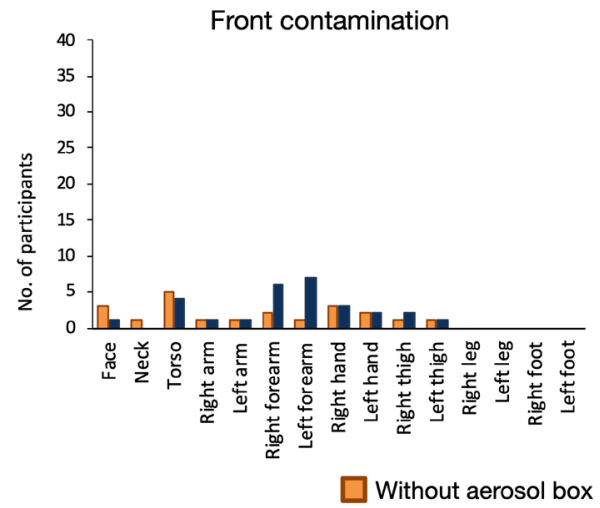

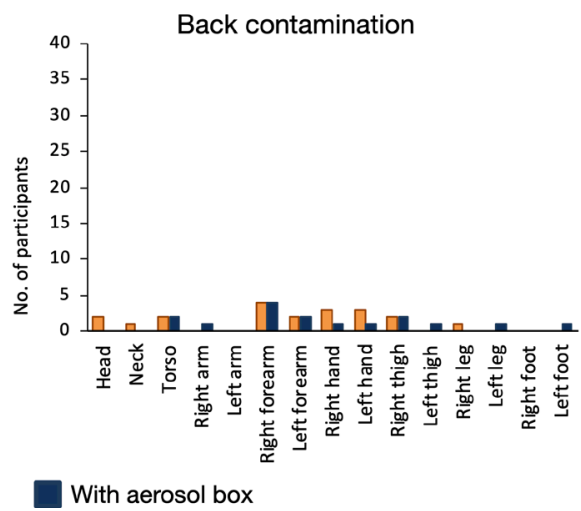

With aerosol box

Figure 3 Frequency of contamination based on body regions of intubators (A) pre-doffing and (B) post-doffing of PPE. McNemar test: * ${ }^{*}<<0.005$, ${ }^{*} \mathrm{p}<0.05$.

Table 3 Participants' subjective rating of their experience in performing intubation with versus without aerosol box

\begin{tabular}{|c|c|c|c|}
\hline & $\begin{array}{l}\text { Without } \\
\text { aerosol box } \\
\mathrm{N}=36\end{array}$ & $\begin{array}{l}\text { With aerosol } \\
\text { box } \\
N=36\end{array}$ & P value* \\
\hline \multicolumn{4}{|l|}{ As intubator, no. (\%) } \\
\hline Handling—easy and very easy & $30(83.3 \%)$ & $25(69.4 \%)$ & 0.267 \\
\hline Visibility—good and very good & $29(80.6 \%)$ & $21(58.3 \%)$ & 0.021 \\
\hline $\begin{array}{l}\text { Confidence—confident and very } \\
\text { confident }\end{array}$ & $31(86.1 \%)$ & $25(69.4 \%)$ & 0.074 \\
\hline Movement-little and no limitation & $31(86.1 \%)$ & $14(38.9 \%)$ & $<0.001$ \\
\hline $\begin{array}{l}\text { Perception of exposure risk—high } \\
\text { and very high }\end{array}$ & $25(69.4 \%)$ & $5(13.9 \%)$ & $<0.001$ \\
\hline \multicolumn{4}{|l|}{ As assistant, no. (\%) } \\
\hline Handling—easy and very easy & $32(88.9 \%)$ & $28(77.8 \%)$ & 0.219 \\
\hline Visibility—good and very good & $30(83.3 \%)$ & $22(61.1 \%)$ & 0.008 \\
\hline $\begin{array}{l}\text { Confidence-confident and very } \\
\text { confident }\end{array}$ & $31(86.1 \%)$ & $27(75.0 \%)$ & 0.289 \\
\hline Movement-little and no limitation & $29(80.6 \%)$ & $16(44.4 \%)$ & 0.002 \\
\hline $\begin{array}{l}\text { Perception of exposure risk-high } \\
\text { and very high }\end{array}$ & $25(69.4 \%)$ & $2(5.6 \%)$ & $<0.001$ \\
\hline
\end{tabular}

Five-point Likert scale rating: handling of equipment (very difficult to very easy), visibility during performing the procedure (very poor to very good), confidence in achieving successful intubation (not confident to very confident), degree of movement (very limited to no limitation) and perception of risk of exposure to contamination (very low to very high).

${ }^{*}$ McNemar-Bowker test. the time to successful intubation and the number of intubation attempts. The trainees in our study took longer to intubate when using the aerosol box. This finding is similar to Begley et al's ${ }^{22}$ study that compared intubation time between no aerosol box and two different aerosol box designs. Handling equipment within the confines of a small box poses a challenge particularly in a difficult airway situation or when adjunct devices are used. In our study, we did not simulate a difficult airway with the airway manikin and the majority of the trainees achieved CormackLehane grade 1. In Begley et al's ${ }^{22}$ study, their airway manikin's tongue was inflated to simulate Cormack-Lehane grade $2 \mathrm{~A}$ and all their anaesthetists elected to use bougie on the first attempt. They found longer intubation time and higher unsuccessful intubation rate as compared with our study findings. Differences in the box design between our study and Begley et al's study may have also accounted for the differences of results.

Although visibility during performing the procedure was rated to be better by the majority of our trainees when intubating without the aerosol box, there was no significant difference in VL-CL score by the intubators between using and not using the aerosol box and also between the intubators and assessor. This may be because of the use of a video laryngoscope with a camera view monitor placed at the side of the bed (and outside of the aerosol box). The results may be different if a video laryngoscope with a handle-mounted display was used.

Our study had several limitations. Even though the Glo Germ solution has particle sizes of $1-5 \mu \mathrm{m}$ and has been used in a previous study as surrogate airway droplet and aerosol contaminants, ${ }^{15}$ it may not accurately represent airborne virus spread 
such as the SARS-CoV-2. It was also not possible to blind the participants to the trajectory of aerosols from the nebuliser chamber. However, Glo Germ is visible only under UVA light in darkened room. To minimise bias, study investigators involved in measuring the contaminations were blinded to the intervention assignment. This was a manikin-based simulation study and involved only few intubation steps. This allowed us to standardise the assessment, but it could only represent a real airway management scenario to a limited extent.

In conclusion, the use of an aerosol box significantly reduced contaminations on the PPE of healthcare workers involved in the intubation procedure. However, this was at the expense of increased intubation time and reduced operator's movement and visibility. Furthermore, the difference in degree of contamination between the use of an aerosol box and no aerosol box could be offset by proper doffing of PPE. Clinicians should make decisions on whether to use the aerosol box with caution, balancing between risks and benefits, particularly in difficult airway situations. Strict adherence to proper donning and doffing of PPE and good hand hygiene should remain the emphasis when performing aerosol generating procedures.

Acknowledgements The authors thank Mohmmad Salleh Yahya, Head of Department of Emergency Medicine, University Malaya Medical Centre, for his administrative support in providing the time and space for us to conduct this study.

Contributors MNA provided the conception and design of the study, analysis, validation and interpretation of data, drafted the manuscript, revised it critically for important intellectual content, and provided final approval of the version to be submitted. AB provided the design of the study and data collection instruments, analysis, validation and interpretation of data, drafted the manuscript and revised it critically for important intellectual content, and provided final approval of the version to be submitted. KP and AZAZ provided the design of the study, coordinated and supervised the acquisition of data, drafted the manuscript and revised it critically for important intellectual content, and provided final approval of the version to be submitted. MZAMN, MAAA, MHMY, AZ, JYOC, AK, BTA and Al supplied the acquisition of data, revised the drafted manuscript critically for important intellectual content, and provided final approval of the version to be submitted. AZ and AK coordinated the blinding of investigators and revised the drafted the manuscript and for important intellectual content. KSC revised the drafted manuscript critically for important intellectual content.

Funding The authors have not declared a specific grant for this research from any funding agency in the public, commercial or not-for-profit sectors.

Competing interests None declared.

Patient consent for publication Not required.

Ethics approval This study was granted ethics approval from the University of Malaya Medical Research Ethics Committee (MREC ID number: 202041-8412). Enrolment of participants was voluntary, and each recruited participant signed an informed consent form.

Provenance and peer review Not commissioned; externally peer reviewed.

Data availability statement Data are available upon reasonable request. The datasets used and/or analysed during the current study are available from the corresponding author on reasonable request.

Supplemental material This content has been supplied by the author(s). It has not been vetted by BMJ Publishing Group Limited (BMJ) and may not have been peer-reviewed. Any opinions or recommendations discussed are solely those of the author(s) and are not endorsed by BMJ. BMJ disclaims all liability and responsibility arising from any reliance placed on the content. Where the content includes any translated material, BMJ does not warrant the accuracy and reliability of the translations (including but not limited to local regulations, clinical guidelines, terminology, drug names and drug dosages), and is not responsible for any error and/or omissions arising from translation and adaptation or otherwise.
Open access This is an open access article distributed in accordance with the Creative Commons Attribution Non Commercial (CC BY-NC 4.0) license, which permits others to distribute, remix, adapt, build upon this work non-commercially, and license their derivative works on different terms, provided the original work is properly cited, appropriate credit is given, any changes made indicated, and the use is non-commercial. See: http://creativecommons.org/licenses/by-nc/4.0/.

\section{ORCID iD}

Aida Bustam http://orcid.org/0000-0001-9403-0734

\section{REFERENCES}

1 Aylward B, Liang W. Report of the WHO-China joint mission on coronavirus disease 2019 (COVID-19), 2020. Available: https://www.who.int/publications/i/item/report-ofthe-who-china-joint-mission-on-coronavirus-disease-2019-(covid-19) [Accessed 31 May 2020].

2 Cook TM. Personal protective equipment during the coronavirus disease (COVID) 2019 pandemic - a narrative review. Anaesthesia 2020;75:920-7.

3 Brewster DJ, Chrimes N, Do TB, et al. Consensus statement: safe airway Society principles of airway management and tracheal intubation specific to the COVID-19 adult patient group. Med J Aust 2020;212:472-81.

4 Cook TM, El-Boghdadly K, McGuire B, et al. Consensus guidelines for managing the airway in patients with COVID-19: guidelines from the difficult airway Society, the association of anaesthetists the intensive care Society, the faculty of intensive care medicine and the Royal College of anaesthetists. Anaesthesia 2020;75:785-99.

5 Bahl P, Doolan C, de Silva C, et al. Airborne or droplet precautions for health workers treating coronavirus disease 2019? J Infect Dis 2020;93.

6 Tran K, Cimon K, Severn M, et al. Aerosol generating procedures and risk of transmission of acute respiratory infections to healthcare workers: a systematic review. PLoS One 2012;7:e35797.

7 van Doremalen N, Bushmaker T, Morris DH, et al. Aerosol and surface stability of HCoV-19 (SARS-CoV-2) compared to SARS-CoV-1. medRxiv 2020. doi:10.1101/2020. 03.09.20033217. [Epub ahead of print: 13 Mar 2020].

8 Coronavirus disease 2019 (COVID-19) pandemic: increased in the EU/EEA and the UK - seventh update, 25 March 2020, 2020. Available: https://www.ecdc.europa.eu/ sites/default/files/documents/RRA-seventh-update-Outbreak-of-coronavirus-diseaseCOVID-19.pdf [Accessed 31 May 2020].

9 Burrer SL, de Perio MA, Hughes MM, et al. Characteristics of health care personnel with COVID-19 - United States. MMWR Morb Mortal Wkly Rep 2020.

10 Lai HY. Aerosol box, 2020. Available: https://sites.google.com/view/aerosolbox/home? authuser $=0$ [Accessed 31 May 2020].

11 Kearsley R. Intubation boxes for managing the airway in patients with COVID-19. Anaesthesia 2020;75:969

12 Canelli R, Connor CW, Gonzalez M, et al. Barrier Enclosure during endotracheal intubation. N Engl J Med 2020;382:1957-8.

13 Li T, Yu T, Hawkins BS, et al. Design, analysis, and reporting of crossover trials for inclusion in a meta-analysis. PLoS One 2015;10:e0133023.

14 Mills EJ, Chan A-W, Wu P, et al. Design, analysis, and presentation of crossover trials. Trials 2009;10:27.

15 Matava CT, Yu J, Denning S. Clear plastic drapes may be effective at limiting aerosolization and droplet spray during extubation: implications for COVID-19. Can J Anaesth 2020;67:902-4.

16 Cohen J. Statistical power analysis for the behavioral sciences. New York: Routledge Academic, 1988.

17 Lee $\mathrm{M}-\mathrm{a}$, Huh K, Jeong J, et al. Adherence to protocols by healthcare workers and selfcontamination during doffing of personal protective equipment. Am J Infect Control 2018;46:S11.

18 Phan LT, Maita D, Mortiz DC, et al. Personal protective equipment doffing practices of healthcare workers. J Occup Environ Hyg 2019;16:575-81.

19 Kwon JH, Burnham C-AD, Reske KA, et al. Assessment of healthcare worker protocol deviations and Self-Contamination during personal protective equipment Donning and Doffing. Infect Control Hosp Epidemiol 2017;38:1077-83.

20 Mumma JM, Durso FT, Ferguson AN, et al. Human factors risk analyses of a Doffing protocol for Ebola-Level personal protective equipment: mapping errors to contamination. Clin Infect Dis 2018:66:950-8.

21 Simpson JP, Wong DN, Verco L, et al. Measurement of airborne particle exposure during simulated tracheal intubation using various proposed aerosol containment devices during the COVID-19 pandemic. Anaesthesia 2020;323.

22 Begley JL, Lavery KE, Nickson CP, et al. The aerosol box for intubation in coronavirus disease 2019 patients: an in-situ simulation crossover study. Anaesthesia 2020;75:1014-21. 